\section{Measurement of social dominance in squirrel monkeys*}

\section{LINCOLN D. CLARK $†$ and EDWIN N. NAKASHIMA ††}

University of Utah College of Medicine, Salt Lake City, Utah 84112 alternative locations available to the animal. Whenever a pair of animals are on the rod, one is invariably higher than the other. This creates a lively interaction between animals as they switch positions to remain highest on the rod. When they reach the top end of the rod, the dominant animal displaces his partner to hold the top position. The behavioral units which are counted in this test are the number

Several laboratory
monkeys are described.

The purpose of this paper is to describe several laboratory methods for measuring competitive in teraction between male squirrel monkeys. Three of these procedures are based upon a characteristic behavior of these arboreal primates, whereby dominant animals preempt perches or cage positions above those of more submissive ones. The fourth procedure involves a more conventional situation, food competition.

The chamber shown in Fig. 1 represents our first efforts to use perch preference as a dominance measure. $A$ pair of animals was placed in the Plexiglas chamber. Since they cannot cling to its smooth walls, they must locate themselves either on one of the perches or on the chamber floor. Using a time sampling procedure, we recorded the position of each monkey at $5 \mathrm{sec}$ intervals for 100 observations. For each sample an animal was given 2 points if on the top perch, 1 point for the lower, and 0 for the floor. An animal moving from one position to another at the moment of observation was scored in the location he was leaving. Total points accumulated after 100 samples provided a "perch score" for each $\mathrm{S}$.

The score produced by this procedure correlated well with other indices of dominance during initial tests of each pair of animals. However, the scores on successive tests over time became increasingly variable, until at times the otherwise obviously dominant animal would obtain a lower perch score than the submissive one. It appeared that the dominant animals, having demonstrated their ability to take the top perch in initial encounters, thereafter were not motivated to repeat the performance and might explore the floor of the cage rather than seek either perch.

Figure 2 represents a situation which provides an inclined rod bridging a cage. The enclosure may be

* From the Department of Psychiatry Behavioral Sciences Laboratory, College of Medicine, University of Utah. This research was supported by NIH Grant MH 13208.

+Professor of Psychiatry, College of Medicine, University of Utah, Research Scientist Awardee, NIH, MH-14,003.

+ Research Associate, Behavioral Sciences Laboratory.

of chicken wire, as illustrated, or preferably of Plexiglas to reduce

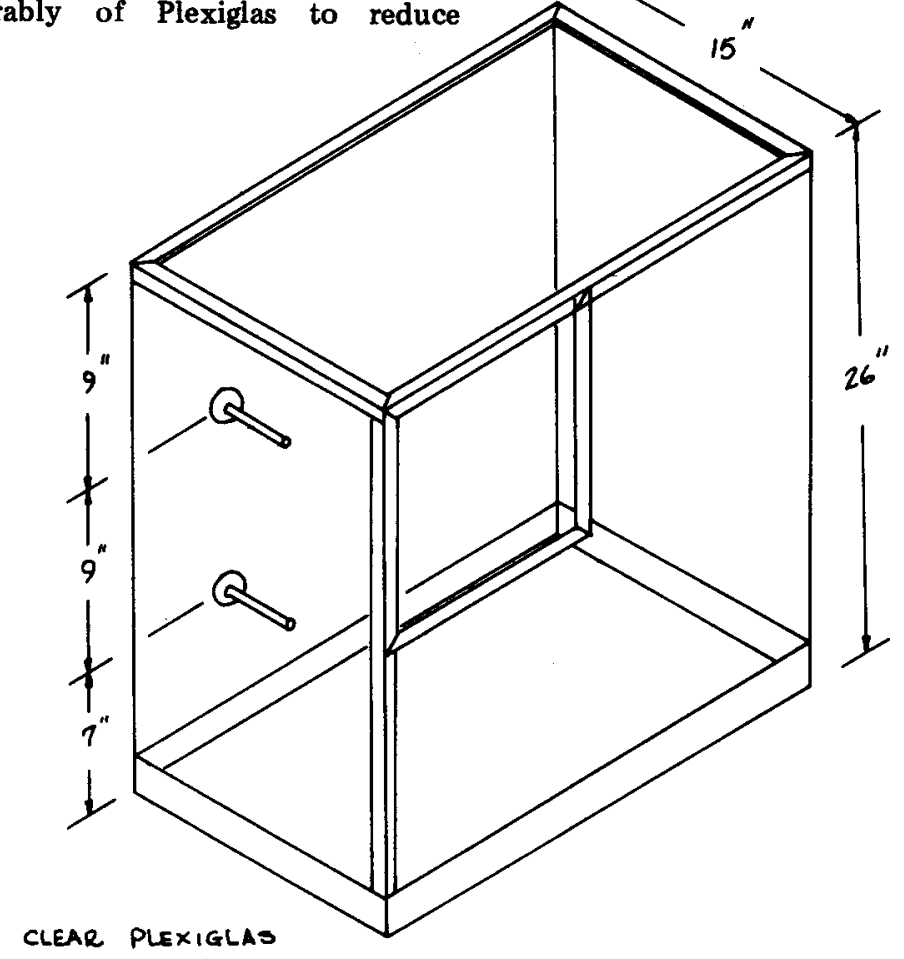

ENCLOSURE

Fig. 1. Perch competition chamber.

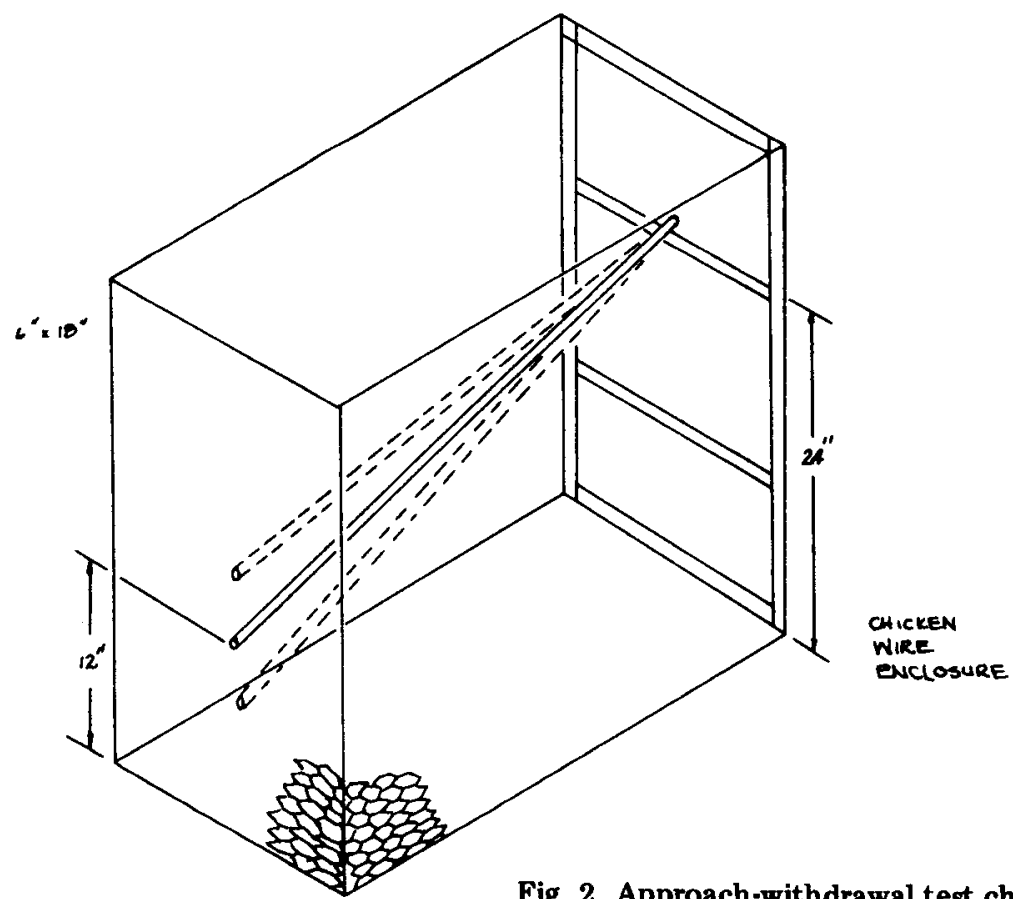

Fig. 2. Approach-withdrawal test chamber. 


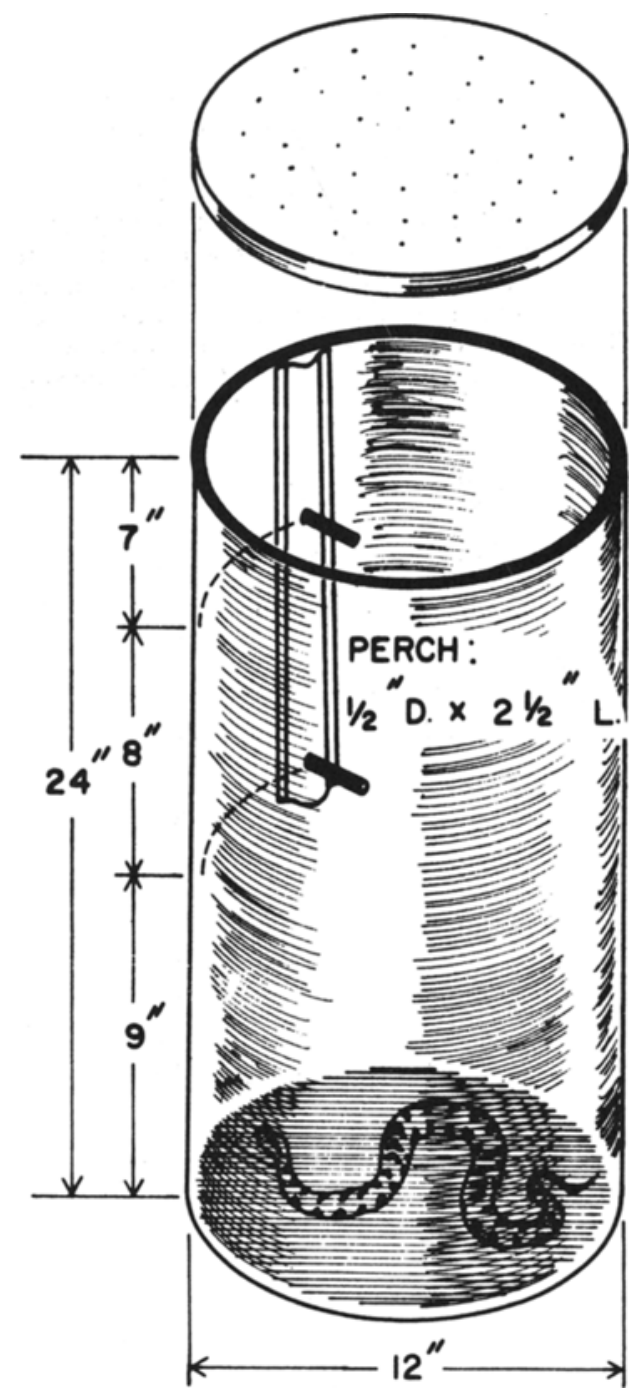

Fig. 3. Perch -escape competition cylinder.

of times the approach of one animal leads to withdrawal of the other. During 100 consecutive 5 sec intervals, the first approach-withdrawal episode to occur within the 5 -sec interval is credited as a score to the initiator. The limitations of these "approachwithdrawal scores" are similar to those of the perch scores described above, i.e., instability on repeated measures, and probably for the same reasons. If the submissive partner no longer attempts to assert himself, the number of aggressive encounters and the score of the dominant animal tends to be smaller. This was reflected in a reliability study in which 20 pairs of animals were given five trials at 3-day intervals with both the perch and approach-withdrawal tests. Both procedures proved stable indicators of dominance through the initial two trials, with a high $(r=0.92)$ correlation between measures. There was a progressive increase in variability through the last three trials. However,

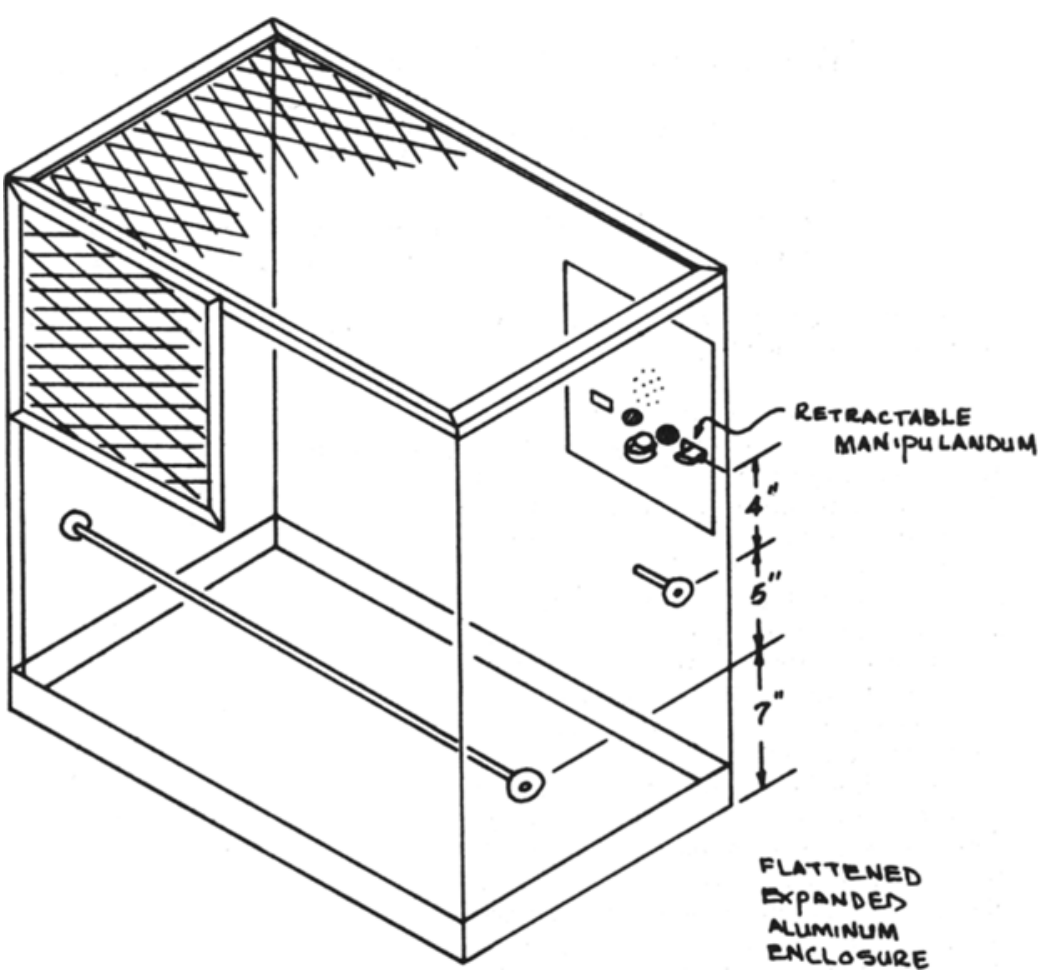

Fig. 4. Operant competition for food.

to rank order groups as large as five in a dominance hierarchy. This is accomplished by pairing individuals in all possible combinations. The percentage of superior positions for each $S$ at all competitive pairings is used to calculate an average superiority score which expresses cardinal dominance of one animal over the others.

Figure 4 shows apparatus used to identify dominance as it is expressed in food competition. It does require that the animals be trained to operate a lever to obtain food pellets. However, this is readily accomplished either for purposes of the test or the investigator may want them so trained for other experiments, as in our case. A pair of animals prepared under equivalent deprivation conditions are placed in the test cage. The test chamber is equipped with an intelligence panel with a retractable lever and pellet dispenser. The retractable lever is extended to initiate a 10-min competitive session. The perch from which the lever can be operated will accommodate only one animal at a time. The percentage of time that an animal monopolizes the manipulandum and consumes the food pellets dispensed represents its score. Although the operant training may seem unnecessarily complex, this approach was adopted after trying a variety of other procedures for presenting food for competition which proved less satisfactory. 